\title{
Prosthetic Complications of Screw-Retained Restoration Using Multiunit Abutments Versus Intraoral Luting on Titanium Base in Implant-Supported Complete Overdentures Randomized Clinical Trial
}

\author{
Mostafa Saeed*(i), Mahmoud Mokhtar El-Far, Amr Hosny Elkhadem \\ Department of Prosthodontics, Faculty of Dentistry, Cairo University, Cairo, Egypt
}

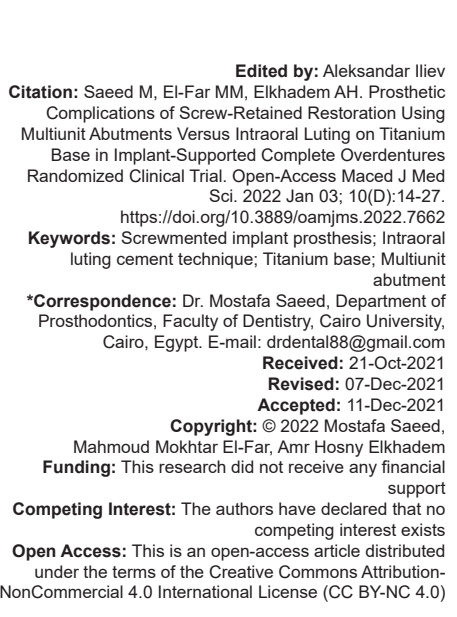

\section{Introduction}

The long-term success of full-arch screwretained implant-supported fixed dental prostheses has become a prime goal in dental prosthetics. As implants became a common line of treatment, structural integrity and long-term durability became one of the main requirements not only to the clinician but also to patients as well. Prosthetic reconstruction involving endosseous implants can include screw-retained or cemented restorations or both together. Although there is no clinically significant difference between the two types, there are ongoing attempts to keep enhancing each category for a better outcome [1]. When dealing with full-arch prostheses, the feature of retrievability is of prime importance to the clinician. Usually, the prosthesis needs an external connection with what is commonly known as a multiunit abutment or a transmucosal abutment to make a screw-retained prosthesis with acceptable passivity and retrievability. Due to the high cost and the need for adequate dental laboratory skills, using a multiunit abutment is not always the treatment of the choice for the clinician in restoring a full-arch dental prosthesis.

The manufacturing revolution in dental implants provides a diverse array of implant accessories that may reduce the cost and also simplify the technical steps required for prostheses construction. Ongoing clinical research aids in refining and updating manufacture to finally provide an easy, durable, and esthetically satisfying outcome for the patient. The titanium bases may seem as a promising solution in the field of customized implant prosthetics. Because of its material and machining, the titanium bases provide superior resistance to fracture and durability for implant-abutment connection. The titanium bases were designed to be compatible with CAD/CAM technology; as it allows faster working 
times and acceptable adaptation [2]. There has been a growing tendency to replace conventional multiunitbased implant prosthetics with titanium base retained prosthetics in an attempt to simplify the prosthetic steps and reduce technical complexities. The use of titanium base retained full-arch restorations is a relatively novel treatment option. The data regarding the long-term (>5 years) [3] stability and prosthetic complications of such treatment are still sparse.

\section{Aim of the study}

The aim of this study was to compare between a full-arch screwmented implant prosthesis using intraoral luting cement technique on titanium bases versus a full-arch screwmented implant prosthesis using transmucosal abutment in terms of prosthetic complication.

\section{Materials and Methods}

\section{Protocol registration}

This trial protocol has been approved by the evidence-based dentistry committee of the department of prosthodontics. All procedures performed in this study involving human participants were in accordance with the ethical standards of the Research Ethics Committee at the Faculty of Dentistry Cairo University Ref (18/10/44). The trial was registered at: ClinicalTrials. gov ID: NCT03674554.

\section{Study settings}

The trial was carried out at Prosthesis department, Faculty of Dentistry - Cairo University and Cairo Implant Academy ( $\mathrm{CI}$ ) research center.

\section{Primary objective}

Patients $(P)$

Completely edentulous patients receiving a full-arch screw-retained implant prosthesis.

\section{Intervention (I)}

Full-arch screw-retained implant prosthesis on titanium bases using intraoral luting cement technique.

\section{Comparator (C)}

Full-arch screw-retained implant prosthesis with transmucosal abutment using intraoral cement technique.
Outcomes (O)

Primary outcome

- $\quad$ Abutment screw loosening.

Secondary outcomes

- $\quad$ Prosthetic screw loosening

- $\quad$ Prosthetic screw fracture

- $\quad$ Abutment screw fracture

- Veneer fracture

- Framework fracture

- Ti-bases decementation

- $\quad$ Overall prosthesis loosening.

\section{Trial design}

This trial was a randomized parallel group controlled trial with an allocation ratio of 1:1.

\section{Eligibility criteria}

\section{Inclusion criteria for participants}

- $\quad$ Completely edentulous patients

- $\quad$ Patients with completely edentulous ridges with opposing natural dentition or implantsupported overdenture

- Completely edentulous patients (with the above-mentioned criteria) who had proper amount of attached gingiva $(\geq 2 \mathrm{~mm}$ )

- Completely edentulous patients (with the above-mentioned criteria) who had no history of bruxism

Completely edentulous patients (with the above-mentioned criteria) who were free or controlled diabetic assessed by measuring glycosylated hemoglobin $\mathrm{A} 1 \mathrm{c}(\mathrm{HbA} 1 \mathrm{c})(\leq 6.4 \%)$.

\section{Exclusion criteria for participants}

- $\quad$ Patients who had a medical condition that absolutely contraindicates implant placement Patients with uncontrolled diabetes, assessed by measuring glycosylated $\mathrm{HbA} 1 \mathrm{c}(>6.4 \%)$ Potentially uncooperative patients who were not willing to go through the proposed interventions. Moderate-to-heavy daily smokers* (who report consuming at least 11 cigarettes/day)

- $\quad$ Patients who had a history of bruxism.

\section{Recruitment}

Patients were selected from the outpatient clinic of the Prosthesis Department, Faculty of Dentistry, Cairo University and CIA research center. Patients who already registered on the department's database were considered for screening and enrollment. All patients 
that fulfilled the previously mentioned inclusion criteria will be included in the study. Screening and enrolment of patients continued until the target population was achieved.

A total of 20 patients were recruited in the trial and divided into nine male and 11 female patients. The ages of the selected participant ranged between 41 and 55 years old. A total of 40 implants in each group were used to construct the full-arch prosthesis for the recruited patients. Four implants were used in each arch.

\section{Allocation}

\section{Randomization}

The allocation ratio was (1:1) and the type of randomization was block randomization (however, the Ti-base group had a dropout of one patient due to failed implants).

\section{Allocation concealment mechanism}

Each participant grasped an opaque sealed opaque envelope from a box at the prosthetic phase. The envelope was opaque and sealed to ensure allocation concealment.

\section{Implementation}

Allocation sequence generation will be performed by MF.

\section{Masking/blinding}

Allocation sequence generation was performed by MF. Enrolment of participants was performed by $M S$ and $A E$ where the patients were assessed for eligibility.

\section{Sample size calculation}

Based on a study conducted by Jacqueline $P$. Duncan, the abutment screw loosening of the control group was reported by $8 \%$ ( $p$. note 0.8 ). Considering equivalent frame with an equivalent limit of $8 \%$, the abutment screw loosening of the intervention group was $16 \%$. The relative risk was 2 . In our RCT with an allocation ratio of $1: 1$, we needed 42 experimental and 42 control samples to reject the null hypothesis at a power of $80 \%$ and a level of significance at $5 \%$. The statistical test used was the Chi-square test. The sample size was increased to a total of 104 implants (52 implants/group) to compensate for a dropout rate of about $15 \%$. The program was PS program version 3.0.43.

\section{Participant timeline (Table 1)}

\section{Intervention}

In this study, full-arch screw-retained prostheses were used to restore completely edentulous patients. The restorations were supported and retained by four implants.

After obtaining a thorough medical and dental history, the following steps were done:

Table 1: Procedural time table

\begin{tabular}{|c|c|c|c|c|c|c|c|c|}
\hline Steps & 0 & $\begin{array}{l}1-3 \\
\text { weeks }\end{array}$ & $\begin{array}{l}4-7 \\
\text { weeks }\end{array}$ & $\begin{array}{l}8-11 \\
\text { weeks }\end{array}$ & $\begin{array}{l}23 \\
\text { weeks }\end{array}$ & $\begin{array}{l}24-26 \\
\text { weeks }\end{array}$ & $\begin{array}{l}6 \text { months after } \\
\text { prosthesis } \\
\text { construction }\end{array}$ & $\begin{array}{l}12 \text { months } \\
\text { after prosthesis } \\
\text { construction }\end{array}$ \\
\hline Pt. diagnosis & $\cdot$ & & & & & & & \\
\hline Enrollment & & . & & & & & & \\
\hline Randomization & & . & & & & & & \\
\hline $\begin{array}{l}\text { Construction of } \\
\text { denture }\end{array}$ & & & . & & & & & \\
\hline $\begin{array}{l}\text { Guide } \\
\text { construction }\end{array}$ & & & . & & & & & \\
\hline $\begin{array}{l}\text { Implant } \\
\text { placement }\end{array}$ & & & & . & & & & \\
\hline $\begin{array}{l}\text { Healing } \\
\text { abutment } \\
\text { placement }\end{array}$ & & & & & . & & & \\
\hline $\begin{array}{l}\text { Prosthesis } \\
\text { construction }\end{array}$ & & & & & & . & & \\
\hline Follow-up & & & & & & & . & . \\
\hline
\end{tabular}

\section{Pre-operative prosthetic preparation}

- $\quad$ Eligible patients who were already wearing complete dentures: The dentures were checked for adaptation, fit, stability, and occlusion.

- $\quad$ Eligible patients who were not wearing upper and lower complete dentures:

Preliminary alginate impressions ${ }^{1}$ using a perforated stock tray were recorded

Impressions were then poured into dental stone $^{2}$ to obtain the diagnostic model. Occlusion blocks were constructed over the diagnostic models, followed by bite registration, try-in, and delivery of upper and lower clear denture ${ }^{3}$

- In the constructed complete denture, holes were created using a fissure bur, in the center of each tooth, which were then filled by gutta-percha. ${ }^{4}$

\section{CBCT scanning 5}

CBCT for the arch of interest with the following precautions:

a) The patient was scanned while wearing the scan appliance in the proper seating position. Images with an improperly seated appliance were not acceptable and were repeated.

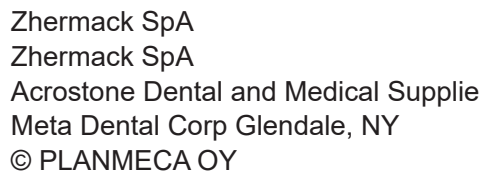


b) Any metallic objects were removed in the head-and-neck region as earrings or glasses.

c) Jaws separation during scan was confirmed by biting over a cotton roll or a similar object.

Teeth positions were identified by the radioopaque gutta-percha fillings which were inserted at the center of each tooth. Implants were added from the software implant library with proper length and width according to the patient's available bone length and width with consideration to the adjacent anatomical structures.

\section{Surgical phase}

Under local anesthesia, ${ }^{6}$ a mid-crestal full arch incision was performed. A buccal full thickness flap was raised.

After proper disinfection using betadine ${ }^{7}$, the radiographic stent was placed in the patient's mouth and a mark was drawn corresponding to the planed implant position, then a palatal/lingual full-thickness flap was raised (Figure 1).

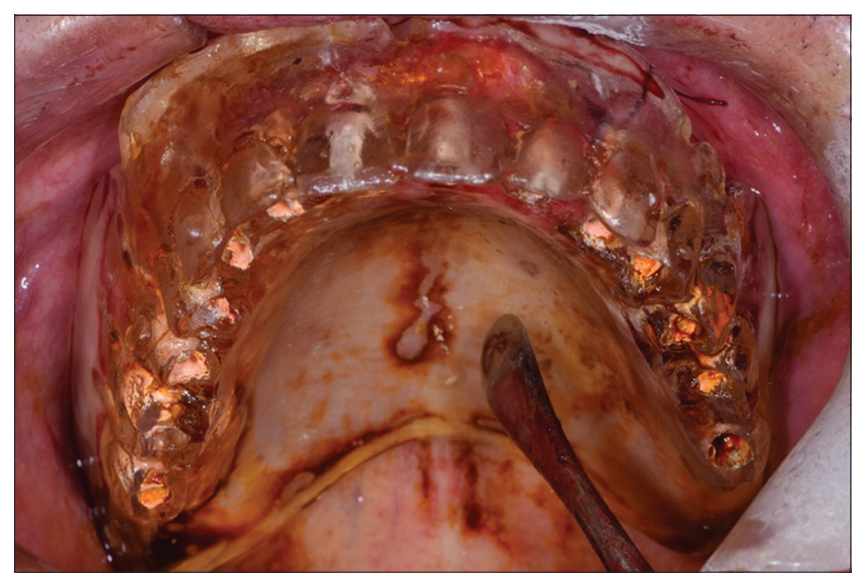

Figure 1: Surgical stent

According to the pre-planned implant position, sequential drilling was made starting with the cortical drill, the pilot drill, intermediate drills, final drill, and finally the countersink drill. Paralleling pins were placed into the osteotomy after the pilot drill to verify parallelism (Figure 2).

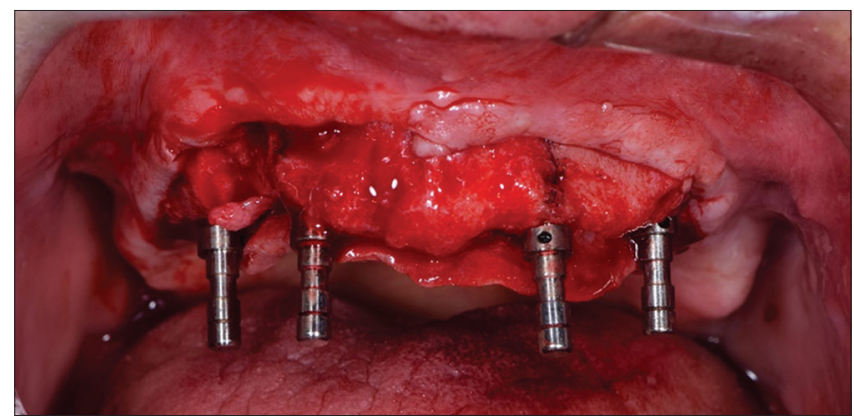

Figure 2: Paralleling pins

6 Artinibsa . Inibsa dental S.L.U . Barcelona . spain

7 Mundipharma Middle East FZ-LLC 2021
In case of angled distal implants, a guide ${ }^{8}$ was used to aid in aligning distal implants.

Bone level endosseous implants with internal conical hex connection ${ }^{9}$ were placed in their corresponding osteotomies and were submerged $1 \mathrm{~mm}$ under bone level. Cover screws were fastened by hand torque. Continuous locked sutures ${ }^{10}$ were made using surgical suture to ensure watertight seal closure.

\section{Prosthetic phase}

The steps for final prosthesis fabrication were initiated 3 months after implant placement for both groups, the prosthetic phase was accomplished for each group as follows:

\section{Implant exposure}

\section{Group 1: Multiunit abutment group}

Under local anesthesia, ${ }^{11}$ using the patient's surgical stent to locate implant position, a small midcrestal incision was made. A flap was raised and implants were uncovered. Cover screws were untied and multiunit abutments were fastened according to manufacturer recommendations at 30 Newtons using a torque wrench. To minimize settling effect, the multiunits were retightened at least twice at the recommended manufacturer torque at a $10 \mathrm{~min}$ interval. Multiunit healing caps were fastened and interrupted sutures were made (if needed).

\section{Group 2: Ti-base group}

Under local anesthesia ${ }^{12}$ using the patient's surgical stent to locate implant position, a small midcrestal incision was made. A flap was raised and implants were uncovered and cover screws were untied. Healing caps were fastened and interrupted sutures were made (if needed).

\section{Impression procedures}

For both groups, primary impressions were made to fabricate a custom tray. The custom tray was characterized by two main options. The first was a relief space to accommodate the acrylic splinting between impression copings. The second was a wide perforation to allow accessibility to the impression coping screws. Patients were left for 10 days to allow gingival healing. Thereafter, sutures were cut away.

\footnotetext{
8 B and B Dental Implant Company

9 DENTIS Co., LTD.

10 (C) Suture Express

11 Artinibsa, Inibsa dental S.L.U., Barcelona, Spain

12 Artinibsa, Inibsa dental S.L.U., Barcelona, Spain
} 


\section{Group 1: Multiunit abutment group}

Healing caps were untied and regular open tray pick-up abutment level impression copings were fastened over multiunit abutments by manual torque.

\section{Group 2: Ti-base group}

Healing caps were untied and regular open tray pick-up implant level impression copings were fastened over multiunit by manual torque (Figure 3 ).

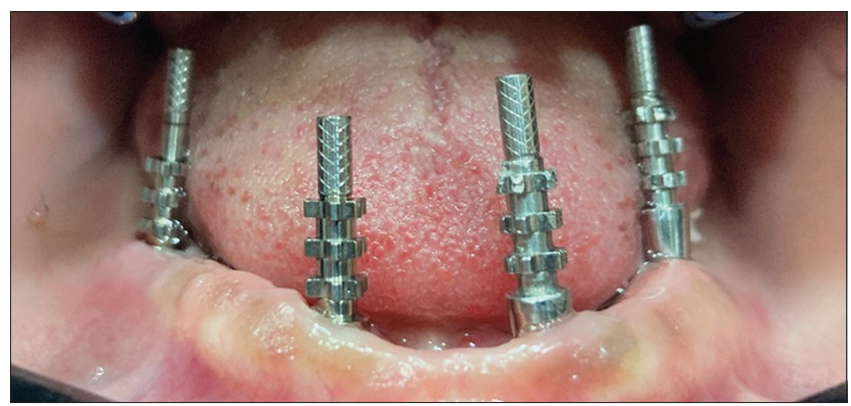

Figure 3: Open tray transfer

\section{Impression pouring}

For both groups, to ensure impression accuracy, splinting of the impression copings was made through self-cure acrylic resin. ${ }^{13}$ A single-stage impression technique (putty and light body) was made using silicon impression material $^{14}$ with the aid of the prefabricated custom tray. The healing caps were refastened after the impression was made. Analogs were assembled over the picked-up impression coping. Tissue mimic ${ }^{15}$ material was utilized on the impression through overlaying the analog impression coping connection. Pouring of the final impression with extra hard dental stone was carried out.

\section{Verification jig construction}

\section{Group 1: Multiunit abutment group}

Over the resulted cast, acrylic verification jig was constructed over the impression coping using a self-cure acrylic resin ${ }^{16}$ to verify the accuracy of the final impression. One screw test was applied to check passivity. If the test revealed a non-passive structure, jig separation and intraoral assembly were performed. The laboratory technician then grinded the cast around the non-passive multiunit analog and reattached it in its new position with dental stone.

\section{Group 2: Ti-base group}

Over the resulted cast, acrylic verification jig was constructed over the Ti-base using self-cure acrylic

13 Duralay, Prestige Dentl Products UK Ltd.

14 Kettenbach $\mathrm{GmbH}$ and $\mathrm{Co}$. KG

15 Multisil mask bredent $\mathrm{GmbH}$ and Co.KG

16 Duralay, Prestige Dentl Products, UK Ltd resin ${ }^{17}$ to verify the accuracy of the final impression. One screw test was applied to check passivity. If the one test revealed a non-passive structure, jig separation and intraoral assemblage were performed. The laboratory technician then grinded the cast around the non-passive multiunit analog and reattached it in its new position with dental stone (Figure 4).

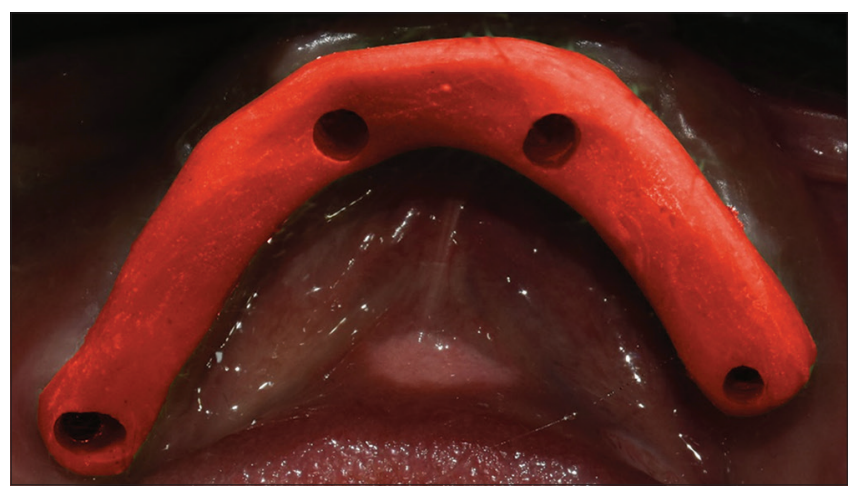

Figure 4: Verification jig

\section{Framework construction}

For both groups, occlusion blocks ${ }^{18}$ were constructed over the final casts for bite registration. After bite registration, the case was ready for scanning, ${ }^{19}$ designing, ${ }^{20}$ nesting, ${ }^{21}$ and milling ${ }^{22}$ (Figure 5).

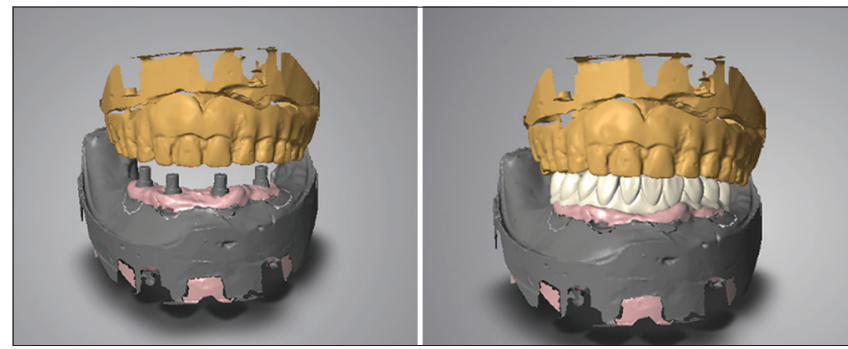

Figure 5: Cast scanning/framework designing

Try-in preparation

Once the milling was finished, the PEEK disk material $^{23}$ was removed from the milling machine. The supporting rods were cut-off and the laboratory started to finish the supporting rods attached to the bases from the milled frame. The frame was seated on the articulator to be checked. Permanent cement was used to attach only one metal cylinder (the technique will be discussed later), temporary cement ${ }^{24}$ was used

17 Duralay, Prestige Dentl Products, UK Ltd

18 Acrostone Dental and Medical Supplies

19 SHERAeco-scan 7 SHERA Werkstoff-Technologie $\mathrm{GmbH}$ and Co. KG

20 DWOS - Dental Wings Inc.

21 SHERAEco-Mill-CAD. SHERA Werkstoff-Technologie GmbH and Co. KG

22 SHERAeco-mill 5x SHERA Werkstoff-Technologie $\mathrm{GmbH}$ and Co. KG

23 Bredent $\mathrm{GmbH}$ and Co.KG

24 Cavex Holland BV 
to attach the remaining three metal cylinders to the PEEK frame, preparing it for the intraoral try-in. The permanent cementation of one single metal cylinder allows for proper seating of the framework and prevents its movement due to tissue rebound in deeply placed implants. The PEEK frame was checked intraorally to verify passivity and occlusion.

\section{Framework veneering}

Following the try-in stage, the framework was ready to go through delivery procedure. The framework was sent to the laboratory to be veneered with acrylic teeth and Visio.lign. ${ }^{25}$ The laboratory carried out the final restoration in the following steps:

1. Setting of the acrylic readymade $\operatorname{teeth}^{26}$ on the milled framework with modeling wax $^{27}$ considering the opposing occlusion.

2. A putty impression material ${ }^{28}$ was applied on the buccal surface of the final setting and on the buccal side of master cast to construct a putty index.

3. Longitudinal grooves were made on the cast to help in putty index repositioning.

4. The putty index was removed and the master cast was soaked in boiling water to dissolve the modeling wax.

5. Retentive grooves were made on the framework and the teeth.

6. Under six bars for $10 \mathrm{~min}$, the framework and the inner surface of the teeth were sandblasted using aluminum oxide particles with a size of $110 \mu \mathrm{m}$.

7. Replacing of the sandblasted teeth in their positions in the putty index.

8. Light cure primer for PEEK and PEKK polymers (Visiolink Primer) ${ }^{29}$ was applied on the sandblasted surfaces (framework and the teeth) using a microbrush then, both the framework and the teeth were placed in a curing box and were left to cure for $90 \mathrm{~s}$.

9. Dual-hardening Luting Composite (Combo. lign $)^{30}$ was added to the teeth and the framework then, the putty index holding the teeth was repositioned in its place.

10. Excess cement was removed and the assembly was placed in the cure box to cure for $3 \mathrm{~min}$.

11. The putty index was detached from the cast, thereafter, the cast with the attached teeth was left in the curing box for $90 \mathrm{~s}$.

12. The cast was left for 15 min to guarantee a selfcure process completion before the finishing step.

Bredent $\mathrm{GmbH}$ and Co.KG

Bredent $\mathrm{GmbH}$ and Co.KG

Cavex Holland BV

Zhermach S.p.A. Italy

Bredent $\mathrm{GmbH}$ and Co.KG

Bredent $\mathrm{GmbH}$ and Co.KG
13. The excess cement was finished using finishing burs.

14. On the gingival area of the prosthesis, sandblasting was performed with the same parameters as the teeth area.

15. Light cure primer for PEEK and PEKK polymers was applied on the framework using a microbrush, then the prosthesis was placed in a curing box and left to cure for $90 \mathrm{~s}$.

16. Using Crea.lign GUM materials $\AA,{ }^{31}$ gingival prosthesis was built to the desired shape, thereafter, the entire prosthesis was placed in the curing box for 6 min.

17. The dispersion layer (oxygen inhibited composite layer) was removed with alcohol, then the prosthesis was ready for delivery step (Figure 6).

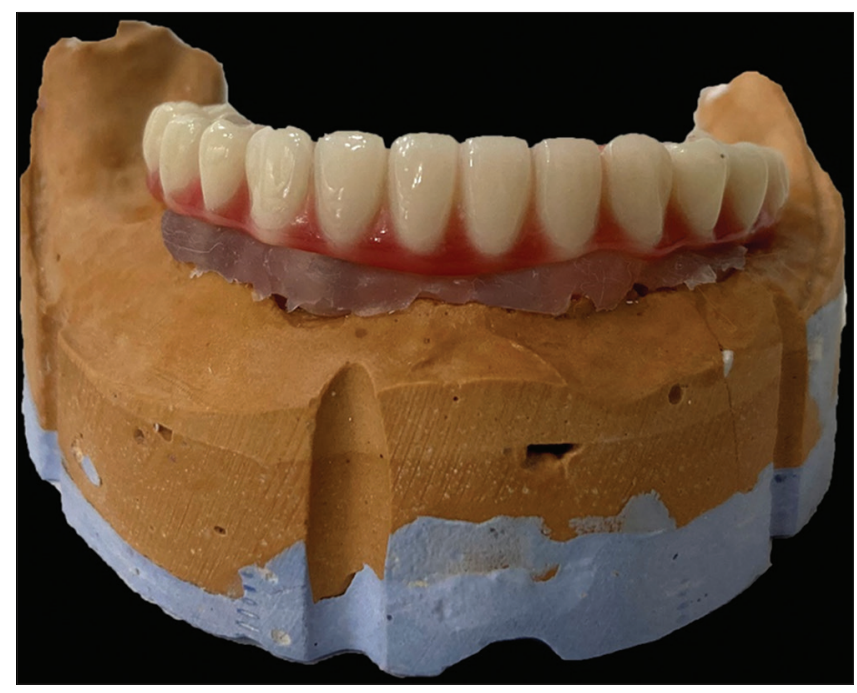

Figure 6: Final prosthesis after veneering

\section{Intraoral cementation procedures}

According to Bredent $\circledast$ instructions, intraoral luting of the prosthesis on the titanium abutment at the delivery stage was done in the following steps:

1. Under six bars for $10 \mathrm{~min}$, the PEEK framework at the abutment area and the metal abutment was sandblasted using aluminum oxide with a particle size of $110 \mu \mathrm{m}$.

2. Light cure primer for PEEK and PEKK polymers (Visiolink Primer®) was applied on the framework using a microbrush, then the framework was placed in curing box and left for $90 \mathrm{~s}$ for to cure.

3. The titanium abutments were fastened intraorally and proper moisture control was achieved using cotton rolls and the suction unit.

4. Metal primer $^{32}$ (MKZ Primer $\circledast$ ) was applied on the sandblasted titanium abutments.

31 Bredent $\mathrm{GmbH}$ and Co.KG

32 Bredent $\mathrm{GmbH}$ and Co.KG 
5. Dual-hardening luting composite (Combo. lign $($ ) was added to the framework.

6. Intraoral seating of the prosthesis was done and intraoral curing ${ }^{33}$ of the luting composite was made.

7. After $20 \mathrm{~s}$ of curing for each abutment, the abutment/prosthetic screws were unfastened and then the prosthesis was removed from the patient's mouth along with the picked-up titanium abutments.

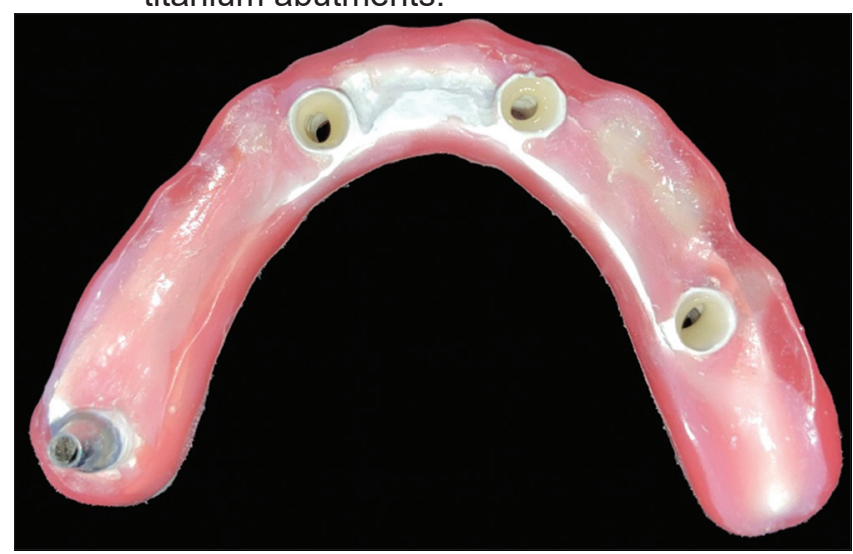

Figure 7: Cement application in the prosthesis

8. Extraoral curing for each abutment was then carried out for $20 \mathrm{~s}$, followed by removal of the excess cement and refastening the prosthesis' screws following the manufacturer's instructions. In case of Group 1, the manufacturer recommends to tighten the prosthetic screw at 20 Newtons. On the other hand, the manufacturer recommends tightening the abutment screws at 30 Newtons.

9. Covering the screw channel was made using cotton piece with overlaying light cured composite (Figures 7 and 8).

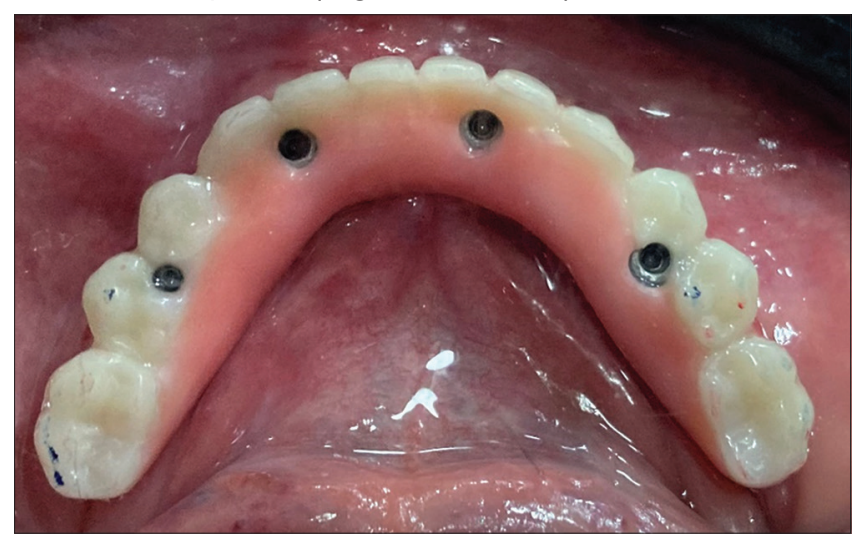

Figure 8: Final cementation

\section{Data collection methods}

$\mathrm{AE}$ - cosupervisor collected the demographic and baseline information from the patients. Data collection forms were designed by the principal investigator (MS) for the collection of outcome data to

33 Guilin Woodpecker Medical Instrument Co., Ltd. ensure data quality. After the data had been collected by MS from all patients, it was pooled, tabulated, and stored with the senior supervisor to initiate analysis. It is important to mention that any data that were documented within the first 6 months would fall under the "6 months data" group, on the other hand, any data that were documented within the second 6 months period would fall under the "12 months data" group. Throughout the exposure process for implants, two implants failed in one patient from the Ti-base group. This patient was excluded from the study.

\section{Data management}

Original paper forms related to study data were entered electronically using an Excel workbook to obtain a soft copy. A monthly data back-up was performed by $A E$ who was totally responsible for the process of data management and scheduling a periodic back-up.

\section{Data monitoring}

\section{Monitoring}

Dr. Amr Elkhadem (AE) was responsible for periodic inspection of the accumulating outcome data and trial conduct; in addition to guidance about modifying or discontinuing the trial.

\section{Stopping guidelines}

The following guidelines were followed for excluding patients from the trial:

- An allergic reaction to titanium.

- Aserious complication after implant placement.

- Any relevant deterioration in the health of the subject possibly affecting participation in the trial.

- $\quad$ Failure of the participant to comply with trial requirements.

Withdrawal of the informed consent.

- $\quad$ More than $40 \%$ implant failure.

\section{Harms}

Regardless of any decision to modify or discontinue their assigned intervention, study participants were retained in the trial whenever possible to enable follow-up data collection and prevent missing data. The trial was discontinued or modified based on a decision taken by the data monitoring committee. If mucositis or bone resorption more than $3 \mathrm{~mm}$ was reported during the follow-up period, a suitable antibiotic and mouthwash will be given to the patient who would then be recalled bimonthly to closely observe the changes. If the mucositis or peri-implantitis persisted, the prosthesis was removed for 2 weeks 
till inflammation subsides. A continuation of bone loss or inflammation dictated trial discontinuation. If the clinician detected a failed implant, proper curettage was done and placement of new implants with a wider diameter or greater length was carried out in the same osteotomy. This procedure was done only if the clinician found four sound walls of bone in the failed site after curettage. If there was deficient bone at the failed site, the clinician packed bone graft material and allowed the site to heal for 6 months without placing an implant. After 6 months, an implant was placed in the available bone. Other reasons for modifying the trial included patient dissatisfaction with the aesthetics or function.

\section{Informed consent}

The principal investigator (MS) obtained informed consents from potential trial participants after explaining the interventions and their possible benefits and risks.

\section{Confidentiality}

All administrative forms, reports, photos, radiographs, and laboratory investigations were kept in locked cabinets at a secure and accessible place. Moreover, they were identified by a coded identification number to ensure participant confidentiality. Electronic data were secured with password protected access systems. Access to the study data was restricted to the principal investigators (MS and $\mathrm{AH}$ ).

\section{Declaration of interest}

There was no conflict of interest present.

\section{Access to data}

Trial investigators (MS, $A E$, and MF) had access to encrypted patients' files.

\section{Post-delivery instructions for the patients}

- $\quad$ Brushing: Used with a soft detergent or a denture paste and water.

- Use of a polishing tooth paste was contraindicated, abrasives could lead to denture wear and stiff bristles could cause obvious abrasion. The fitting surface should not be brushed too aggressively.

- $\quad$ Cut fruits into small pieces prior to eating.

- $\quad$ Annual check-ups were essential for denture patients (every 6 months)

- $\quad$ Any complications or unusual situation as prosthesis loosening, veneer fracture, and pain necessitate immediate contact with the doctor.

\section{Reporting outcomes}

Outcomes were reported as binary data for each patient in Table 2. Visual inspection for the prosthesis was done without any intervention in case of absence of signs or symptoms indicating complications. If either visual inspection or patient complaints revealed any prosthetic complication, the clinician acted accordingly with the proper action as shown in Table 3:

Table 2: Incidence of prosthetic complications

\begin{tabular}{|c|c|c|c|c|}
\hline \multirow[t]{2}{*}{ Complications } & \multicolumn{2}{|l|}{6 months } & \multicolumn{2}{|c|}{12 months } \\
\hline & Ti-bases & Multiunit & Ti-bases & Multiunit \\
\hline Abutment screw loosening (by implants) & 0 & 0 & 4 & 0 \\
\hline Prosthetic screw loosening (by implants) & $\mathrm{N} / \mathrm{I}$ & 0 & $\mathrm{~N} / \mathrm{I}$ & 8 \\
\hline Prosthetic screw fracture (by implants) & $\mathrm{N} / \mathrm{l}$ & 0 & $\mathrm{~N} / \mathrm{I}$ & 1 \\
\hline Abutment screw fracture (by implants) & 0 & 0 & 0 & 0 \\
\hline Veneer fracture (by cases) & 2 & 2 & 1 & 2 \\
\hline Framework fracture (by cases) & 0 & 0 & 0 & 0 \\
\hline Ti-bases decementation (by implants) & 4 & $\mathrm{~N} / \mathrm{l}$ & 7 & $\mathrm{~N} / \mathrm{l}$ \\
\hline Overall prosthetic loosening (by cases) & 1 & 0 & 3 & 2 \\
\hline
\end{tabular}

\section{Statistical methods}

Data were explored for normality using Kolmogorov-Smirnov and Shapiro-Wilk tests. The data showed non-parametric (not-normal) distribution. Qualitative data were presented as frequencies and percentages. Wilcoxon and Mann-Whitney tests were used to compare the qualitative outcomes in this study. The significance level was set at $p \leq 0.05$. Statistical analysis was performed with IBM $\AA$ SPSS $₫$ Statistics Version 20.

Table 3: Management of prosthetic complications

\begin{tabular}{ll}
\hline Complication & Treatment \\
\hline Overall prosthesis loosening (due to screw loosening) & Screw retightening \\
Prosthetic/abutment screw fracture & Screw retrieval then \\
& Screw replacement \\
Veneer fracture (within three teeth) & Intraoral reveneering \\
Veneer fracture (more than 3 teeth) & Lab. reveneering \\
Framework fracture & Prosthesis remake \\
Ti-bases decementation & Intraoral recementation \\
\hline
\end{tabular}

\section{Results}

Data were explored for normality using Kolmogorov-Smirnov and Shapiro-Wilk tests, data showed non-parametric (not-normal) distribution.

Qualitative data were presented as frequencies and percentages. Wilcoxon and Mann-Whitney tests were used to compare the qualitative outcomes in this study. The significance level was set at $P \leq 0.05$. Statistical analysis was performed with IBM $®$ SPSS $®$ Statistics Version 20 (Tables 2-4).

\section{Abutment screw loosening}

\section{Effect of groups}

$6 \mathrm{~m}$ : 
Table 4: Number of cases allocated to each group

\begin{tabular}{llll}
\hline Ti-base & & Multiunit & \\
\hline 9 cases & & 10 cases & \\
36 implants & & 40 implants & \\
\hline Male & Female & Male & Female \\
\hline 4 & 5 & 4 & 6
\end{tabular}

There was no statistically significant difference between (Ti-base) and (multiunit) groups where $(p=1)$.

- $12 \mathrm{~m}$ :

There was no statistically significant difference between (Ti-base) and (multiunit) groups where $p=$ 0.310 .

\section{Effect of time}

- Ti-base:

There was no statistically significant difference between $6 \mathrm{~m}$ and $12 \mathrm{~m}$ groups where $\mathrm{p}=0.050$.

- Multiunit:

There was no statistically significant difference between $6 \mathrm{~m}$ and $12 \mathrm{~m}$ groups where $\mathrm{p}=1$.

\section{Prosthetic screw loosening}

Effect of groups

- $6 \mathrm{~m}$ :

There was no statistically significant difference between Ti-base and multiunit groups where $p=1$.

\section{- $12 \mathrm{~m}$ :}

There was no statistically significant difference between Ti-base and multiunit groups where $p=0.150$.

\section{Effect of time}

- Ti-base:

There was no statistically significant difference between $6 \mathrm{~m}$ and $12 \mathrm{~m}$ groups where $\mathrm{p}=1$.

- Multiunit:

There was no statistically significant difference between $6 \mathrm{~m}$ and $12 \mathrm{~m}$ groups where $\mathrm{p}=0.150$.

\section{Prosthetic screw fracture}

\section{Effect of groups}

\section{- $6 \mathrm{~m}$ :}

There was no statistically significant difference between Ti-base and multiunit groups where $p=1$.

$12 \mathrm{~m}$ :

There was no statistically significant difference between Ti-base and multiunit groups where $p=0.343$.

\section{Effect of time}

- Ti-base:
There was no statistically significant difference between $6 \mathrm{~m}$ and $12 \mathrm{~m}$ groups where $\mathrm{p}=1$.

- Multiunit:

There was no statistically significant difference between $6 \mathrm{~m}$ and $12 \mathrm{~m}$ groups where $\mathrm{p}=0.317$.

\section{Abutment screw fracture}

Effect of groups

- $6 \mathrm{~m}$ :

There was no statistically significant difference between Ti-base and multiunit groups where $p=1$.

- $12 \mathrm{~m}$ :

There was no statistically significant difference between Ti-base and multiunit groups where $p=1$.

\section{Effect of time}

- Ti-base:

There was no statistically significant difference between $6 \mathrm{~m}$ and $12 \mathrm{~m}$ groups where $\mathrm{p}=1$.

- Multiunit:

There was no statistically significant difference between $6 \mathrm{~m}$ and $12 \mathrm{~m}$ groups where $\mathrm{p}=1$.

\section{Veneer fracture}

\section{Effect of groups}

- $6 \mathrm{~m}$ :

There was no statistically significant difference between Ti-base and multiunit groups where $p=0.908$.

\section{- $12 \mathrm{~m}$ :}

There was no statistically significant difference between Ti-base and multiunit groups where $p=0.606$.

\section{Effect of time}

- Ti-base:

There was no statistically significant difference between $6 \mathrm{~m}$ and $12 \mathrm{~m}$ groups where $\mathrm{p}=0.317$.

- Multiunit:

There was no statistically significant difference between $6 \mathrm{~m}$ and $12 \mathrm{~m}$ groups where $\mathrm{p}=1$.

\section{Framework fracture}

\section{Effect of groups}

$6 \mathrm{~m}:$

There was no statistically significant difference between Ti-base and multiunit groups where $p=1$.

- $12 \mathrm{~m}$ :

There was no statistically significant difference between Ti-base and multiunit groups where $p=1$. 


\section{Effect of time}

\section{- Ti-base:}

There was no statistically significant difference between $6 m$ and $12 m$ groups where $p=1$.

- Multiunit:

There was no statistically significant difference between $6 m$ and $12 m$ groups where $p=1$.

\section{Ti-bases decementation}

Effect of groups

- $6 \mathrm{~m}$ :

There was no statistically significant difference between Ti-base and multiunit groups where $p=0.310$.

- $12 \mathrm{~m}$ :

There was no statistically significant difference between Ti-base and multiunit groups where $p=0.400$.

\section{Effect of time}

\section{- Ti-base:}

There was no statistically significant difference between $6 \mathrm{~m}$ and $12 \mathrm{~m}$ groups where $\mathrm{p}=0.083$.

- Multiunit:

There was no statistically significant difference between $6 \mathrm{~m}$ and $12 \mathrm{~m}$ groups where $\mathrm{p}=1$.

\section{Overall prosthetic loosening}

This outcome was denoted as any clinical event of screw loosening whether abutment screw loosening or prosthesis screw loosening.

\section{Effect of groups}

- $6 \mathrm{~m}$ :

There was no statistically significant difference between Ti-base and multiunit groups where $p=0.292$.

\section{- $12 \mathrm{~m}$ :}

There was no statistically significant difference between Ti-base and multiunit groups where $p=0.521$.

\section{Effect of time}

\section{- Ti-base:}

There was no statistically significant difference between $6 \mathrm{~m}$ and $12 \mathrm{~m}$ groups where $\mathrm{p}=0.157$.

\section{- Multiunit:}

There was no statistically significant difference between $6 \mathrm{~m}$ and $12 \mathrm{~m}$ groups where $\mathrm{p}=0.157$.

\section{Discussion}

In this study, the author aimed to assess the differences between a full-arch screw-retained implant prosthesis on titanium bases using intraoral luting cement technique versus a full-arch screw-retained implant prosthesis with transmucosal abutment in terms of prosthetic complications.

The proper pre-operative planning for the treatment ensures predictable results and fewer complications. To place implants to construct a fullarch implant overdenture prosthesis, it is necessary to perform a trial with set artificial teeth. The try-in stage provides the clinician with numerous diagnostic data regarding prosthesis, esthetics, and function. It is also important to perform what is called a prosthodontically driven implant placement concept. Patients always have concerns regarding the success of implant prostheses in terms of esthetics and function, which are assured by a proper treatment plan [4].

The conversion of the trial teeth setting to a scan appliance with a specified protocol helps to correlate teeth position to the patient's anatomy. It is important to produce a patient radiograph that provides the clinician with an accurate pre-posed implant position that helps to perform the treatment plan. In this study, the principal investigator duplicated the patients' try-in or pre-constructed denture into a scan appliance which was fabricated using self-cure clear acrylic resin. Thereafter, he made a hole through the scan appliance in the center of each tooth and filled it with gutta-percha. Finally, the clinician could correlate the center of each tooth with the underlying patients' anatomy which aids in selecting the proper implant dimension and position. The use of the scan appliance as a surgical stent helped in obtaining an accurate implant position. It was important to raise only the buccal flap before surgical stent seating to guarantee proper seating for the stent [5].

It's well known that the more parallel the implant superstructure, the easier it is to construct full arch implant overdentures. This is why placing parallel pins before implant placement was mandatory. Moreover, the guide used to place angled distal implants helped in placing the implants in the required angle according to the proposed plan. Thus, after placing the angled multiunit, we gained a parallel titanium cylinder [6]. It is important to mention that placing angled distal implants in a few cases are not considered as a confounder in the study. On the contrary, limiting the study to paralle implant fixtures might not standardize the cantilever length due to anatomical variation. Cantilever length was considered as a strong confounder as it might lead to more prosthetic complications [7].

Patients' interarch space was measured during pre-operative planning. Proper prosthetic space allows 
for proper framework thickness thus decreases the incidence of framework and veneer fracture. If the preoperative plan revealed a low interarch space, bone plateauing was required and the implants were placed deeper. On the contrary, if the pre-operative plan revealed high interarch space, removable prosthesis on metal bar was the prosthesis of choice that prosthetic option led to case exclusion [8].

In this study, many steps were made to ensure prosthesis passivity so the total load applied on the prosthesis, cement and the implant components were from the patient's masticatory force. Splinting the impression copings, using additional silicon impression material in a custom tray, construction of a verification jig, and intraoral cementation were all construction steps that helped in creating a passive prosthesis [9].

The first researcher to introduce the concept of splinting of the impression copings during impression procedures using a rigid material was Branemark. He used splinting to stabilize and prevent the rotational, horizontal, and vertical movement of the impression copings. Autopolymerizing acrylic resin was the most common material that has been used to act as a splinting material. Some clinicians splinted the copings directly to the custom tray. However, according to Mojon et al. and Hsu et al., this material has the disadvantage of dimensional instability. They concluded that the autopolymerizing acrylic resin can shrink up to $7.9 \%$ in the first $24 \mathrm{~h}, 80 \%$ of this shrinkage appears within the first $17 \mathrm{~min}$ of mixing. Furthermore, the greater the mass of the acrylic resin, the greater the resultant shrinkage. Therefore, while taking impressions in this study, the clinician minimized the mass of splinting resin material. Furthermore, he sectioned the fabricated acrylic resin splint between the copings with a thin bur and then rejoined the sectioned pieces together again with a small amount of the self-cure acrylic resin (Duralay®) [10], [11].

The most commonly described impression materials for implant cases were addition silicone and polyether, this could be correlated to their accuracy. The implant impression material should have an appropriate resiliency, not to be distorted by removal from undercuts. At the same time, the impression material should be rigid enough to prevent the movement of the implant components during handling and cast pouring procedure. Sorrentino et al. concluded that both addition silicone and polyether impression materials have comparable accuracy [12].

The customized tray could provide an impression with uniform thickness unlike stock trays making it better and more adaptable, especially in implant cases. Burns et al. found that impressions taken with stock trays were significantly less accurate compared to impressions taken using a customized tray. They concluded that the impression material was uniformly distributed in a customized tray and it was more rigid than a stock tray [13].
A verification jig is a commonly used tool to verify the accurate three-dimensional relationship of the implants and serves as a guide to correct imperfect casts. Many materials have been used for the fabrication of verification jigs as aluminum strips, light polymerizing resins, and self-polymerizing resins. De La Cruz et al. stated that the dimensional accuracy provided by the verification jigs was as same as standard impression procedures. Therefore, they suggested that jig fabrication does not evolve the accuracy of stone casts. However, these findings were in contrast with Ercoli et al. one. They stated that the fabrication of a verification jig guaranteed a clinically passive fit for metal frameworks in a full-arch fixed implant-supported complete denture. Ercoli et al. explained that the contrariness by De La Cruz et al. study was an in vitro study that used an experimental layout that represented an ideal setting for implant impression procedures including the parallel implants, on a stone model and three externally hexed implants. Moreover, they clarify that in a clinical scenario, the impression transfer engages the implant at variable subgingival depths, therefore, diminishing the available surface of the impression transfer that can be engaged by the impression material [14], [15].

PEEK is a semi-crystalline linear polycyclic aromatic polymer. It became an important highperformance thermoplastic material for replacing metals in implant superstructures. PEEK is a white, radiolucent, rigid material with high thermal stability up to $335.8^{\circ} \mathrm{C}$. It is non-allergic and has low plaque affinity if polished well. Compared to metals utilized in dentistry, PEEK is more aesthetic, stable, biocompatible, and lighter. However, due to its grayish-brown color, PEEK is not convenient for monolithic aesthetic restorations in anterior teeth. More esthetic material like Visio. lign ${ }^{\circledR}$ should be used as a coating to get an esthetic result. Due to the complex chemical structure and poor wetting ability of PEEK, it is hard to prepare its surface to raise bond strength with composites. The process is technique sensitive and requires a highly professional, well-trained clinician to perform proper bonding to peek material. It is interesting to say that veneer fracture and prosthetic decementation are mainly due to this complex feature [16], [17].

The framework try-in aimed to check passivity and occlusion before final veneering was carried out to acquire better prosthetic seating. It was recommended to permanently cement one of the titanium abutments. The other abutments were temporarily cemented in the laboratory. The tissue rebound movement, especially in implant level cases, may prevent proper seating. It is interesting to mention that tissue rebound forces may cause decementation of the temporary cement if there is no permanent cementation in any abutment. As mentioned before, it is hard to prepare PEEK surface to raise bond strength so, it was important to have a firm commitment to manufacturer instruction about PEEK veneering. In this study, the laboratory committed to 
manufacturer instruction except for the index material. It was recommended to be clear silicon to be able to transmit light. The laboratory used putty impression silicon due to financial issues. To prevent incomplete curing, the laboratory increased the curing time before and after removal of the putty index [18].

The use of conventional methods for constructing a screw-retained prosthesis was mainly based on using a multiunit abutment either angled or straight to allow proper seating and passivity of the prosthesis. The convergences of multiunit abutments' walls that may reach up to $45^{\circ}$ make it easier to find a common path of insertion for multiple implants with angle difference up to $90^{\circ}$. Many factors might control clinician decisions and lead to angled dental implants as anatomical limitations, bone availability, and decrease cantilever length in the planned prosthesis. The principal concept in multiunit abutment was to turn the internal connection into an external connection. The introduction of such a design with an angle option up to $45^{\circ}$ allows for wide-angle correction and more options for aligning multiple angled implants. However, using multiunit abutment in some situations might complicate the treatment and increasing the restoration cost. In case of limited interarch space, using multiunit will reduce the available space by $2 \mathrm{~mm}$ at least. Moreover, the multiunit cost is almost as equal to the fixture cost itself, as well as the variety of prosthetic accessories required to deal with multiunits [19].

The titanium bases appeared as a tool to custom the implant suprastructure without jeopardizing implantabutment connection stability. The titanium bases were designed to be compatible with CAD/CAM technology; it allows faster working times and perfect adaptation, creating a hybrid concept in abutments, in which it can be used as a screw-retained and cement-retained system. The hybrid concept of titanium bases offers the main advantage over cement-retained restoration, that, it can be unfastened after being intraorally cemented to remove any excess cement, thus avoiding possible tissue inflammation that could be induced by the cement, and after this, it can be fastened on the implant. Moreover, titanium bases overmaster the multiunit abutment in its disadvantages regarding the cost and the exploited space. Heller et al. conducted a study using straight transmucosal abutments restored by crowns cemented to titanium bases while in another group placing the crowns directly onto the transmucosal abutment. The study concluded that both types of restorations were viable treatments that did not represent a statistically significant difference [20]. Titanium base designs play a major role in their uses. Implants with internal connection offer titanium bases with internal protrusion into the connection. The titanium base connection may vary in design from a simple $45^{\circ}$ ledge to a hole cone connection with an anti-rotational feature. Its design determined its uses. For instance, a single crown on a titanium base requires a titanium base with a full fit and an anti-rotational feature. On the other hand, a full arch with non-parallel implants favors a simple $45^{\circ}$ ledge to accommodate various implants angle. It is interesting to say that titanium bases with a non-engaging cone connection will permit only a difference in implant angulation equal to the value of double the titanium base cone angle. Internal cone connection angle may vary according to different manufacturing from $1.5^{\circ}$ up to $16^{\circ}$. It seems that a small $45^{\circ}$ ledge may permit perfect seating for a prosthesis on more than 2 implants with difference in angulation, nevertheless, it appears to overload the abutment screw. Although there is no clinical investigation to prove, the cone design may help in implant-abutment connection stability through increasing the surface connection between abutment and implant [21], [22].

It is important to understand the engineering background of our implant and prosthetic components to be able to explain our results. Implant abutment screw is not an accurate description of what we use. The Machinery's Handbook clarifies that bolts are used to assemble unthreaded objects (abutments), typically with the use of a nut (internal implant threading). A screw is usually going through a tapped hole unless it is a self-tapping screw that fabricates its thread. Screws do not need nuts, as they become fastened by being tightened into the hole with a screwdriver or a driver bit which fits into the drive recess. Generally speaking, screws are shorter than the width of the material they are being screwed into so that they do not emerge onto the other side. Bolts are invented to be installed with a suitable nut. The hole for a bolt is not tapped as the bolt is thrust through and is fixed and tightened using a nut at the back of the material being fastened. A bolt will be longer than the width of the material it is being utilized on, as it needs to thrust through to the other side to screw into the nut. The most evident way of differentiating between a bolt and a screw is that a bolt is not usually threaded along its shank as it has a plain portion. A screw, however, is threaded along the shank to the head. Bolts are typically used to create a bolted joint using a nut (internal implant threading) to apply force while using the shank to act as a dowel [23].

Preload is the tension created in a bolt when it is tightened. This tensile force in the bolt generates a compressive force in the bolted joint known as clamp force. For practical purposes, the clamping force in an unloaded bolted joint is supposed to be equal and opposite of the preload. If proper preload, and thus clamp force, is not created or maintained, a variety of problems may arise such as fatigue failure, joint separation, and self-loosening from loading which can fault the bolted joint. According to the manufacture recommendations, it is obvious that an abutment screw can be loaded with a higher clamping force than a prosthetic screw. Mohammed et al. and Yeo et al. found that the length of the abutment screws has no significant influence on screw loosening. On screening their procedures, 
both researchers load different screws in lengths with nearly equal loads. Thus, screw diameter plays the main role in creating a proper preload in the structure (prosthetic screw diameter $1.3 \mathrm{~mm}$ and abutment screw diameter $1.9 \mathrm{~mm}$ ). This finding explains the higher incidence of prosthetic screw loosening in the multiunit group [24], [25], [26], [27].

Although prosthetic screws loosen more, it is noticeable that the overall prosthesis loosening incidence was increased in the Ti-base group. Many causes could explain this phenomenon. As mentioned before, because of the complex chemical structure and poor wetting ability of PEEK, it is hard to prepare its surface to elevate bond strength and bonding with composites. Moreover, in intraoral luting technique on implant level, it is hard to maintain a moisture controlled field for some time, especially in deep implants thus, affecting cement bond strength. Switching the connection from implant level to abutment level makes it easier to control the field as the connection becomes supragingival. Furthermore, the metal sleeve over the multiunit usually binds the PEEK framework at the screw channel in its full length. Making wide surface area for composite-PEEK connection increases the bond strength. On the contrary, Ti-base length is 4-5 $\mathrm{mm}$ according to the manufacture. This short length limits the surface bonding area which affects the cement bonding strength [21], [24].

Usually, abutment or prosthetic screw fracture is not a common event. There was a report of one prosthetic screw fracture in this study. It is important to mention that fracture occurred during screw retightening. It was a fatigue phenomenon.

\section{Conclusion}

Based on the previous study, it can be concluded that:

1. When constructing screw-retained implant prostheses, using either multiunit or titanium base is considered effective.

2. There was no statistically significant difference between the multiunit group and the Ti-base group in terms of prosthetic complications.

3. The choice between multiunit and Ti-base is a decision that must be made according to the situation in each specific case. Clinicians should take into account implants' type, position, angulations, and available restorative space.

\section{Recommendations}

1. Further clinical studies are required with a longer follow-up time.
2. Further clinical studies are required to evaluate the applicability and clinical efficiency of in-lab luting for full-arch implant-supported prosthesis with PEEK framework.

3. Further clinical studies are required to test different Ti-base designs to find out the advantages and disadvantages of each type.

\section{References}

1. Hamed MT, Mously HA, Alamoudi SK, Hashem AB, Naguib GH A systematic review of screw versus cement-retained fixed implant supported reconstructions. Clin Cosmet Investig Dent. 2020;12:9-16. http://doi.org/10.2147/CCIDE.S231070 PMid:32021476

2. Arce C, Lawson MS, Liu P, Chee MS, Lin P, Givan DA. Titanium bases following different surface treatments. Int J Oral Maxillofac Implants. 2018;33(3):530-5. http://doi.org/10.11607/jomi.5915 PMid:29355855

3. Werbelow L, Weiss M, Schramm A. Long-term follow-up of fullarch immediate implant-supported restorations in edentulous jaws: A clinical study. Int J Implant Dent. 2020;6(1):34. http://doi. org/10.1186/s40729-020-00232-8

PMid:32728859

4. Vercruyssen M. The use of CT scan based planning for oral rehabilitation by means of implants and its transfer to the surgical field :Acritical reviewonaccuracy. J Oral Rehabil. 2008;35(6):45474. http://doi.org/10.1111/j.1365-2842.2007.01816.x PMid:18429973

5. Agliardi E, Clericò M, Ciancio P, Massironi D. Immediate loading of full-arch fixed protheses supported by axial and tilted implants for the treatment of edentulous atrophic mandibles. Quintessence Int (Berl). 2010;41(4):285-93. PMid:20305862

6. Ahuja S, Egbert N, Jain V, Cagna D. Managing maxillary proclination with novel designed angulated implants. J Indian Prosthodont Soc. 2017;17(2):202-6. http://doi. org/10.4103/0972-4052.203196 PMid:28584423

7. Elkhadem. The effect of cantilever length and implant tilt on prosthetic and supporting structure in screw retained full arch prosthesis. cairo J. Published online 2011.

8. Aparicio C, Perales P, Rangert B. Tilted implants as an alternative to maxillary sinus grafting: A clinical, radiologic, and periotest study. Clin Implant Dent Relat Res. 2001;3(1):39-49. http://doi.org/10.1111/j.1708-8208.2001.tb00127.x

PMid: 11441542

9. Mahmoud M, Norsiah B, Yunus B. Passive fit in screw retained multi-unit implant prosthesis understanding and achieving: A review of the literature. J Indian Prosthodont Soc. 2014;14(1):16-23. http://doi.org/10.1007/s13191-013-0343-x PMid:24604993

10. Mojon P, Oberholzer JP. Polymerization shrinkage of index and pattern acrylic resins. J Prosthet Dent. 1999;64(6):684-8. http:// doi.org/10.1016/0022-3913(90)90296-o

PMid:2079675

11. Hsu C, Stein RS. A comparative analysis of the accuracy of implant transfer techniques. J Prosthet Dent. 1993;69(6):588-93. http://doi.org/10.1016/0022-3913(93)90287-x

PMid:8320644 
12. Sorrentino R, Gherlone EF, Calesini G. Effect of implant angulation, connection length, and impression material on the dimensional accuracy of implant impressions: An in vitro. Clin Implant Dent Relat Res. 2010;12(Suppl 1):63-76. http://doi. org/10.1111/j.1708-8208.2009.00167.x PMid: 19438937

13. Burns J, Palmer R, Howe L, Wilson R. Accuracy of open tray implant impressions: An in vitro comparison of stock versus custom trays. J Prosthet Dent. 2003;89(3):250-5. http://doi. org/10.1067/mpr.2003.38

PMid:12644799

14. Ercoli C, Geminiani A, Feng C, Lee H. The influence of verification jig on framework fit for nonsegmented fixed implant-supported complete denture. Clin Implant Dent Relat Res. 2012;14(Suppl 1):188-195. http://doi. org/10.1111/j.1708-8208.2011.00425.x PMid:22176765

15. La Cruz JE, Funkenbusch PD, Ercoli C, Moss ME, Graser GN, Tallents RH. Verification jig for implant-supported prostheses: A comparison of standard impressions with verification jigs made of different materials. J Prosthet Dent. 2002;88(3):32936. http://doi.org/10.1067/mpr.2002.128070 PMid:12426505

16. Sulaiman TA. Materials in digital dentistry a review. J Esthet Restor Dent. 2020;32(2):171-81. http://doi.org/10.1111/ jerd. 12566 PMid:31943720

17. Jivraj S, Rawal S. Material Considerations for Full-arch ImplantSupported Restorations. United States: Springer International Publishing; 2018. p. 189-211.

18. Yilmaz B, Kale E, Johnston WM. Marginal discrepancy of CADCAM complete-arch fi xed implant-supported frameworks. J Prosthet Dent. 2018;120(1):65-70. http://doi.org/10.1016/j. prosdent.2017.11.021 PMid:29475755

19. Uludag B, Celik G. Fabrication of a cement and screwretained multiunit implant restoration. J Oral Implantol.
2006;32(5):248-50. http://doi.org/10.1563/811.1

PMid:17069169

20. Heller H, Arieli A, Beitlitum I, Pilo R, Levartovsky S. Load-bearing capacity of zirconia crowns screwed to multi-unit abutments with and without a titanium base: An in vitro pilot study. Materials (Basel). 2019;12(19):3056. http://doi.org/10.3390/ma12193056 PMid:31547045

21. Ladino LG. Titanium bases in implant dentistry: A comprehensive narrative review. Sci Arch Dent Sci. 2020;3:51-9.

22. Ribeiro CG, Maia ML, Scherrer SS, Cardoso AC, Wiskott HW Resistance of three implant-abutment interfaces to fatigue testing. J Appl Oral Sci 2011;19:413-20.

23. Oberg E, Jones FD, Horton HL, Ryffel HH. Machinery's handbook. In: Guide to Machinery's Handbook. 27th ed. New York: Industrial Press; 2004

24. Siamos G, Winkler S, Boberick KG. Relationship between implant preload and screw loosening on implant-supported prostheses. J Oral Implantol 2002;28(2):67-73. http://doi. org/10.1563/1548-1336(2002)028<0067:TRBIPA>2.3.CO;2 PMid: 12498448

25. Mohammed HH, Lee J, Bae J, Cho H. Effect of abutment screw length and cyclic loading on removal torque in external and internal hex implants. J Adv Prosthodont. 2016;8(1):62-9. http:// doi.org/10.4047/jap.2016.8.1.62 PMid:26949489

26. Kim K, Kim M, Kwon H, Lee J. Variation in the total lengths of abutment/implant assemblies generated with a function of applied tightening torque in external and internal implant abutment connection. Clin Oral Implants Res. 2010;22(8):8349. http://doi.org/10.1111/j.1600-0501.2010.02063.x PMid:21198900

27. Yeo IS, Lee JH, Kang TJ, Kim SK, Heo SJ, Koak JY, et al. The effect of abutment screw length on screw loosening in dental implants with external abutment connections after thermocycling. Int J Oral Maxillofac Implants. 2014;29(1):59-62. http://doi.org/10.11607/jomi.3125

PMid:24451854 